\title{
Extending Horizons of Extracorporeal Membrane Oxygenation: Can the Tight Rope of Anticoagulation Help Further?
}

\author{
Santanu Kumar Bora ${ }^{1}$ Ravi Narayan Sahu ${ }^{1}$ \\ ${ }^{1}$ Department of Neurosurgery, All India Institutes of Medical \\ Sciences, Bhubaneswar, Odisha, India
}

J Card Crit Care TSS 2018;2:49-50

We read with great interest the editorial on "Anticoagulation during ECMO: Will the Tight Rope Be Tighter in 2018?" and the various articles of the journal highlighting different recent aspects and significance of extracorporeal membrane oxygenation (ECMO). ${ }^{1}$ We would like to take your attention to a few unconventional indications of ECMO and henceforth discuss the upcoming challenges of anticoagulation on ECMO.

Extracorporeal membrane oxygenation or cardiopulmonary bypass with hypothermic circulatory arrest was used in the past for treatment of neurosurgical patients with giant intracranial aneurysms, but this therapy fell out of favor due to complications associated with coagulopathy and hemorrhage. Traditionally, many centers have also considered suspected or confirmed traumatic brain injury (TBI) as a contraindication to the use of ECMO, given the risk of intracranial hemorrhage (ICH). However, recently, several institutions have described the use of ECMO in elective neurosurgery patients and also in patients with $\mathrm{TBI}^{2}{ }^{2}$ With the given advances in ECMO technology and novel anticoagulation management approaches as mentioned in the editorial, TBI as a contraindication for ECMO should be questioned. ${ }^{1}$ On the other hand, when an ICH complicates extracorporeal support, ECMO is strongly recommended to be discontinued, and, in many cases, no neurosurgical intervention is offered. We, however, believe that neurosurgical intervention, including craniotomy and hemorrhage evacuation, may be considered for well-selected patients with ICH while on ECMO. Anticoagulation management in this setting, however, continues to be controversial. As mentioned in the editorial, we are of the opinion that point-of-care testing with thromboelastography and/or rotational thromboelastometry with platelet aggregometry can be of immense help to the physician as there is an upcoming role for surgical evacuation of hemorrhage in well-selected patients on ECMO in spite of the substantial surgical risk.

\begin{abstract}
Address for correspondence Santanu Kumar Bora, MS, MCh (Neurosurgery), Department of Neurosurgery, All India Institutes of Medical Sciences, Bhubaneswar, Odisha, India (e-mail: dr.santanubora@gmail.com).
\end{abstract}

In the setting of craniotomy, major concern with ECMO therapy is the increased risk of ICH from anticoagulation. When an ICH occurs during ECMO treatment, the physician must balance the risk of hemorrhagic progression against that of ECMO circuit clotting and the ensuing secondary embolic events. There have been many strategies to decrease the need of use of anticoagulation drugs such as heparin in such settings so that ECMO can be instituted in a craniotomy patient after cardiopulmonary collapse without significant intracranial bleeding or neurological sequelae. Chen et al used a heparin-bonded ECMO circuit for a patient after a decompressive craniectomy and avoided the need for systemic heparin. ${ }^{3} \mathrm{~A}$ recent report by Bruzek et al demonstrated a similar lifesaving use of ECMO and systemic heparin in a patient with acute respiratory distress syndrome following a trauma craniectomy. ${ }^{4}$ In the editorial, the use of bivalirudin has been suggested in such setting. ${ }^{1}$ We would like to highlight that a few studies have shown that nafamostat mesilate, a synthetic protease inhibitor, which has been used widely as an anticoagulant for hemodialysis patients, can be used as an alternative anticoagulant to heparin to reduce bleeding complications during ECMO. ${ }^{5}$ Hwang et al have reported the use of ECMO with nafamostat mesilate as the anticoagulant in a patient with ruptured intracranial aneurysm and neurosurgical operation was performed under ECMO therapy. ${ }^{5}$ However, Lim et al have failed to show the beneficial effect of the drug. ${ }^{6}$

In conclusion, we believe that patients with TBI can be supported on ECMO, but extreme precaution must be taken regarding anticoagulation. The newer strategies of anticoagulation and the advances in coagulation monitoring can help in such lifesaving neurosurgical intervention during ECMO support that would extend the horizons of ECMO.

\section{Conflict of Interest}

None.
DOI https://doi.org/ 10.1055/s-0038-1669377. ISSN 2457-0206.
Copyright @2018 Official Publication of The Simulation Society (TSS), accredited by International Society of Cardiovascular Ultrasound (ISCU).
License terms

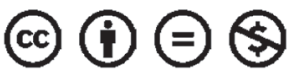




\section{References}

1 Kapoor PM. Anticoagulation during ECMO: will the tight rope be tighter in 2018? J Card Crit Care TSS 2017;1:55-56

2 Muellenbach RM, Redel A, Küstermann J, et al. [Extracorporeal membrane oxygenation and severe traumatic brain injury. Anaesthesist 2011;60(7):647-652

3 Chen YS, Ko WJ, Lin FY, Huang SC, Wang SS, Tu YK. New application of heparin-bonded extracorporeal membrane oxygenation in difficult neurosurgery. Artif Organs 2001;25(8):627-632

4 Bruzek AK, Vega RA, Mathern BE. Extracorporeal membrane oxygenation support as a life-saving measure for acute respiratory distress syndrome after craniectomy. J Neurosurg Anesthesiol 2014;26(3):259-260

5 Hwang GJ, Sheen SH, Kim HS, et al. Extracorporeal membrane oxygenation for acute life-threatening neurogenic pulmonary edema following rupture of an intracranial aneurysm. J Korean Med Sci 2013;28(6):962-964

6 Lim JY, Kim JB, Choo SJ, Chung CH, Lee JW, Jung SH. Anticoagulation during extracorporeal membrane oxygenation; nafamostat mesilate versus heparin. Ann Thorac Surg 2016;102(2):534-539 